\title{
Eine Pathoanalyse der Religion
}

\author{
Bemerkungen zu einem unvollendeten Projekt Freuds
}

Herman Westerink

Zusammenfassung: Eine Grundannahme der Freud'schen psychoanalytischen Methodik ist die Idee, dass die Psychoneurosen als Vergrößerung und Verdichtung normaler, allgemeinmenschlicher Psychodynamiken und Komplexe betrachtet werden können und dass daher diese Normalität am besten aus Sicht der Psychopathologie erforscht werden kann (Pathoanalyse). In diesem Aufsatz wird die Bedeutung der Pathoanalyse für die Erforschung der Religion rekonstruiert und untersucht; es wird auch gezeigt, warum Freud ein solches Projekt nur ansatzweise durchgeführt hat.

Abstract: An important premise in Freud's psychoanalytic methodology is the idea that the psychoneuroses are magnifications and intensifications of normal generally human psychic dynamics and complexes, and that consequently human nature can best be studied from the perspective of the psychopathologies (patho-analysis). In this article the importance and implications of this principle for the study of religion are explored. Also, the article identifies some of the main reasons why Freud never consequently pursued the patho-analysis of religion.

\section{Einleitung}

In seinem Text „Psychische Behandlung (Seelenbehandlung)“ aus dem Jahre 1890 schreibt Sigmund Freud: „Erst wenn man das Krankhafte studiert, lernt man das Normale verstehen". "Diese Bemerkung ist paradigmatisch für eine methodische Vorgangsweise, die er während seines Lebens immer wieder artikulieren und anwenden wird. In seinen „Neuen Vorlesungen“ von 1933 wird Freud diese Vorgangsweise mit der Metapher des Kristalls beschreiben:

„Das Ich ist also spaltbar, es spaltet sich während mancher seiner Funktionen, wenigstens vorübergehend. Die Teilstücke können sich nachher wieder vereinigen. Das ist gerade keine Neuigkeit, vielleicht eine ungewohnte Betonung allgemein bekannter Dinge. Anderseits sind wir mit der Auffassung vertraut, daß die Pathologie uns durch ihre Vergrößerungen und Vergröberungen auf normale Verhältnisse aufmerksam machen kann, die uns sonst entgangen wären. Wo sie uns einen Bruch oder Riß zeigt, kann normalerweise eine Gliederung vorhanden sein. Wenn wir einen Kristall zu Boden werfen, zerbricht er, aber nicht willkürlich, er zerfällt dabei nach seinen Spaltrichtungen in Stücke, deren Abgrenzung, obwohl unsichtbar, doch durch die Struktur des Kristalls vorherbestimmt war. Solche rissige und gesprungene Strukturen sind auch die Geisteskranken.“"

1 Sigmund Freud, Psychische Behandlung (Seelenbehandlung), in: Studienausgabe Ergänzungsband, Berlin 1989, 20.

2 Ders., Neue Folge der Vorlesungen zur Einführung in die Psychoanalyse, in: Studienausgabe, Bd. I, Berlin 1989, 497-498. 
Die Kernidee dieser Passage, die man das Kristallprinzip nennen kann, ist dieselbe, wie sie 1890 schon zum ersten Mal formuliert worden war, nämlich der Grundgedanke, dass die Pathologie als Vergrößerungen und Vergröberungen normaler psychischer Strukturen uns eben gerade deswegen die Gelegenheit bietet, solche normalen Strukturen, „die uns sonst entgangen wären“, zu erkennen und zu analysieren. Anders gesagt: Durch die Pathologie kann man das Mensch-Sein überhaupt verstehen. Die Psychoanalyse wird so zu einer Anthropologie. Diesen Anspruch finden wir explizit in jenem Text Freuds, der wahrscheinlich das beste Beispiel für diese methodische Vorgangsweise und ihre Implikationen darstellt, nämlich in den „Drei Abhandlungen zur Sexualtheorie" in der Originalfassung von 1905, wo Freud bezüglich seiner Vorgangsweise bemerkt, dass "die pathologischen Gesichtspunkte von anthropologischen abgelöst worden " sind. ${ }^{3}$ Er meint damit nichts anderes, als dass seine klinischen Einsichten zu Hysterie und Perversion nicht auf pathologische Abirrungen beschränkt bleiben sollten, sondern eine anthropologische Relevanz haben, insofern durch diese Einsichten die menschliche Sexualität im Allgemeinen beschrieben werden kann. In der Tat wird in den Drei Abhandlungen die Hysterie zum Hauptparadigma für die weitere Konzeptualisierung von Sexualität. Freud möchte Hysterie bzw. Perversion nicht als Resultat abnormer neuropathischer Veranlagung erklären und als eigenständige Entität verstehen, sondern von einer allgemein menschlichen sexuellen Konstitution ausgehen. In der Hysterie finden wir nämlich anlagebedingt überdurchschnittlich hohe sexuelle Energie und in der Folge eine Verdrängung von Sexualtrieben, die „über das normale Maß hinausgeht“. Hysterie ist eine Erkrankung, die, wie Freud behauptet, dem Normalen zumindest nahesteht und durch das gleichzeitige Vorliegen dreier Merkmale charakterisiert ist: durch ein Übermaß an sexueller Energie, durch gesteigerte und exzessive Verdrängung und durch körperliche Symptombildungen, die als Vergrößerungen normaler körperlicher Äußerungen des stets mehr oder weniger instabilen emotionalen Lebens in den Blick kommen. In diesem Sinne fungiert Hysterie gleichsam als Vergrößerungsglas zur Analyse allgemein menschlicher Dynamiken.

Freud geht noch einen bedeutenden Schritt weiter: Das menschliche Leben kann deswegen am besten aus der Perspektive einer bestimmten Gruppe von Erkrankungen (nämlich den Psychoneurosen) untersucht werden, weil diese Erkrankungen auf Steigerungen normaler physiologischer und psychischer Prozesse und Mechanismen hinweisen und im Verhältnis zu anderen Erkrankungen nicht sonderlich stark von der Normalität abweichen. Wenn wir alle zu

3 Ders., Drei Abhandlungen zur Sexualtheorie (1905), in: Studienausgabe, Bd. V, Berlin 1989, 51. Die Erstfassung der „Drei Abhandlungen“ wurde 1905 bei Deuticke (Wien \& Leipzig) publiziert. Eine ausführlich kommentierte Neu-Ausgabe ist 2015 erschienen: ders., Drei Abhandlungen zur Sexualtheorie (1905), Philippe Van Haute/Christian Huber/Herman Westerink (Hg.), Sigmund Freuds Werke - Wiener Interdisziplinäre Kommentare, Bd. 2, Wien 2015.

4 Ders., Drei Abhandlungen zur Sexualtheorie, 73. 
einem gewissen Grad hysterisch sind, kann uns Hysterie darüber Aufschluss geben, wer wir sind. ${ }^{5}$ Anhand dieses Freud'schen Gedankengangs lässt sich die Einführung der Hysterie als Paradigma für die Untersuchung der Sexualität in den „Drei Abhandlungen“ begreifen. Daher erweist sich Hysterie als tragfähig für eine anthropologische Herangehensweise an die Sexualität und damit ans Mensch-Sein. Die Bausteine der menschlichen Sexualität können am besten und vielleicht sogar nur auf Grund der Analyse der Psychoneurosen aufgedeckt werden. Aus der Untersuchung von Erkrankungen entsteht das, was man die "Pathoanalyse der menschlichen Existenz" nennen könnte. ${ }^{6}$ Die menschliche Natur kann am besten - und wahrscheinlich sogar ausschließlich - aus der Perspektive psychopathologischer Variationen untersucht werden.

Diese pathoanalytische Perspektive hat in der Literatur erstaunlich wenig Aufmerksamkeit erregt. Und dennoch ist sie fundamental, um die Ansprüche und Besonderheiten der Freud'schen Psychoanalyse überhaupt verstehen zu können. Welche Ansprüche und Besonderheiten? Erstens geht es natürlich um die gerade erwähnte Wende zum allgemein Menschlichen; sie wird erst durch die Prämisse der pathoanalytischen Perspektive möglich: Die Psychopathologien sollte man nicht als eigenständige Dispositionen darstellen, die der normalen menschlichen Disposition als Abirrung oder Defekt gegenüber stehen, sondern man soll beide innerhalb eines Kontinuums betrachten. Zweitens wird erst durch diese Perspektive die angewandte Psychoanalyse ermöglicht. Dies wird explizit thematisiert von Otto Rank und Hans Sachs in ihrem programmatischen Aufsatz zur angewandten Psychoanalyse, der 1912 die erste Nummer der Zeitschrift Imago eröffnete.

\section{Pathoanalyse und angewandte Psychoanalyse}

Nach Rank und Sachs hat Freud selbst die Möglichkeit entdeckt, wie seine Erkenntnisse zur Entstehung und zum Aufbau der Psychoneurosen auf Vorgänge und Erzeugnisse des normalen Seelenlebens angewendet werden können:

„Die erste Überraschung und Nötigung zur Selbstbesinnung erlebte die Psychoanalyse mit der unerwarteten Entdeckung, daß alle jene seelischen Eindrücke und Vorgänge sich bei normal gebliebenen und selbst zu besonders hohen Leistungen befähigten Menschen im gleichen Ausmaße nachweisen ließen. Die hysterische Konversion war nur ein Zerrbild dessen, was wir alle als den normalen körperlichen

5 Ebd., 74.

6 Siehe dazu: Philippe Van Haute, Psychoanalysis and/as Philosophy. The Anthropological Significance of Pathology in Freud's Three Essays on the Theory of Sexuality and in the Psychoanalytic Tradition, in: Natureza Humana 7 (2), 359-374; ders./Tomas Geyskens, A Non-Oedipal Psychoanalysis? A Clinical Anthropology of Hysteria in the Work of Freud and Lacan, Leuven 2012; ders./Herman Westerink, Hysterie, Sexualität und Psychiatrie. Eine Relektüre der ersten Ausgabe der „Drei Abhandlungen zur Sexualtheorie“, in: Freud, Drei Abhandlungen zur Sexualtheorie (1905), Wien 2015, 9-56. 
Ausdruck der Gemütsbewegung empfinden, die Verdrängung unlustbetonter Vorstellungen ist uns allen im Beiseiteschieben unerwünschter Erinnerungen und ihrem Erfolg, dem Vergessen, geläufig. [...] Das Seelenleben des Gemütskranken zeigt uns diese wie alle anderen seelischen Erscheinungen nur in krasser Übertreibung, und erleichtert uns eben damit den Einblick in die Mechanik des gesamten seelischen Geschehens. Waren so die rätselhaften ,Nervenkrankheiten', denen man bisher durch scharfe Kontrastierung zum Normalen verständnislos und überlegen lachend gegenüberstand, durch ihre Annäherung an normales Geschehen dem Verständnis näher gebracht worden, so warfen andererseits die pathologisch verzerrten Vorgänge im Neurotiker ein klärendes Licht in die dunklen Tiefen unserer Seelenvorgänge."

Der nächste Schritt ist die logische Folge dieses Kontinuums zwischen Pathologie und Normalität: „Von der Einzelpsyche aber läßt sich unschwer der Übergang zu dem Verständnis der Kulturformen als Niederschlag des Zusammenwirkens zahlloser Einzelseelen finden“. ${ }^{8}$ Wenn also kulturelle Phänomene als Produkte von zu Kollektiven zusammengeschweißten Individuen betrachtet werden können, so kann die Psychoanalyse ihre pathoanalytische Perspektive von der Anthropologie hin zur Kulturtheorie erweitern. Aber welche kulturellen Phänomene würden sich hier für psychoanalytische Erforschung eignen? Rank und Sachs erwähnen drei, nämlich Mythos, Religion und Kunst, die alle drei als „Durchbruchsgebilde“ und „Bühnen“ für „die im praktischen Kulturleben unverwertbar gewordenen mächtigen Triebregungen " gesehen werden können. ${ }^{9}$ Diese letzte Bemerkung ist besonders wichtig, denn sie macht implizit deutlich, warum gerade Mythos, Religion und Kunst sich als Forschungsgegenstände anbieten. Alle drei sind Phänomene, die nicht rein funktional zu deuten oder erklären sind: Mythos, Religion und Kunst werden in allen Kulturen hoch geschätzt, dienen aber nicht der Selbst- oder Arterhaltung. Sie können also nicht funktional aus den beiden Triebströmen - Sexualtrieb und Nahrungsbedürfnis - erklärt werden. Diese drei kulturellen Phänomene sind daher als Durchbruchsgebilde für verdrängte und unverwertbare Triebe zu qualifizieren, allerdings in dem Sinn, dass in diesen Phänomenen die unverwertbaren Triebe nicht auf asoziale Weise zur Vorschein kommen, sondern sich in sozial angepassten Leistungen zeigen. Gerade deswegen werden auch Mythos, Religion und Kunst so hoch geschätzt: Die nicht verwertbaren Triebregungen werden über diese kulturellen Phänomene abgeführt, ohne dass das $\mathrm{zu}$ individuellen und gesellschaftlichen Problemen führt. Als Beispiel kann man hier an Freuds Aufsatz über „Zwangshandlungen und Religionsübungen“ denken: Den aggressiven Triebregungen wird in der Religion eine Bühne geboten in der Phantasie, es gäbe einen Gott, der sich an meiner Stelle rächen wird an demjenigen, den ich hasse. ${ }^{10}$ Diese Durch-

7 Otto Rank/Hans Sachs, Entwicklung und Ansprüche der Psychoanalyse, in: Imago 1, 1-16 (4).

8 Ebd., 1.

9 Ebd., 13.

10 Freud, Zwangshandlungen und Religionsübungen, in: Studienausgabe, Bd. VII, Berlin 1989, 21. 
bruchsgebilde als Bühnen für sonst unverwertbare Triebregungen kann man also nicht funktional erklären (sie liefern keinen Beitrag zur Art- und Selbsterhaltung), sind aber von zentraler Bedeutung für die Stabilität einer Gesellschaft und haben nur in diesem Sinn sogar eine gesellschaftliche Funktion.

Es ist wichtig zu erkennen, wie Rank und Sachs in der zitierten Passage die Begriffe, Zerrbild' und, Verzerrung' verwenden. Die Hysterie ist kein Zerrbild normaler seelischer Vorgänge in dem Sinne, dass die Hysterie dem normalen Seelenleben gegenüberstünde und davon getrennt wäre, sondern diese Begriffe deuten auf die ,krasse Übertreibung' hin, d.h. die Vergrößerung und Vergröberung normaler psychischer Vorgänge. Der Begriff ,Zerrbild' ist wichtig in Hinblick auf die Lektüre von Freuds „Totem und Tabu“, besonders in einer zentralen Passage:

„Die Neurosen zeigen einerseits auffällige und tiefreichende Übereinstimmungen mit den großen sozialen Produktionen der Kunst, der Religion und der Philosophie, andererseits erscheinen sie wie Verzerrungen derselben. Man könnte den Ausspruch wagen, eine Hysterie sei ein Zerrbild einer Kunstschöpfung, eine Zwangsneurose ein Zerrbild einer Religion, ein paranoischer Wahn ein Zerrbild eines philosophischen Systems. ${ }^{\text {"11 }}$

In Vergleich zu Rank und Sachs hat Freud den Mythos weggelassen und stattdessen die Philosophie eingefügt. Dies ermöglicht ihm, die drei wichtigsten Psychoneurosen und deren zentrale Komplexe mit den unterschiedlichen kulturellen Phänomenen in Verbindung zu setzen. Die Passage kann auf folgende Art interpretiert werden: Wenn man die Kunst verstehen will, so kann man diese am besten aus der pathoanalytischen Perspektive erforschen, indem man die Hysterie als ,Muster' nimmt; wenn man die Religion verstehen will, so kann man diese am besten erforschen, indem man die Zwangsneurose als ,Muster' nimmt, und wenn man philosophische Systeme verstehen möchte, so kann man am besten die paranoische Wahnbildung als Muster nehmen. Was bedeutet das? Oder: was bringt eine solche Analyse?

Nehmen wir als Beispiel den paranoischen Wahn in Beziehung zu den philosophischen Systemen. Freud sagt eigentlich nichts über diese Beziehung, und in seinen Werken ist eine Pathoanalyse der Philosophie kein weiter verfolgtes Thema. Aber was würde uns eine solche Analyse bringen? Gewiss kann man hier, aufgrund von Freuds Aussagen über den Wahn, Elemente rekonstruieren. Erstens, der Wahn - so wissen wir aus der Fallstudie zu Schreber - ist ein ,Heilungsversuch', die Rekonstruktion einer Beziehung zu den Objekten und Dingen in der Welt, nachdem in der psychotischen Krise die Libido von diesen Objekten und Dingen abgelöst worden war. ${ }^{12}$ Dieser Heilungsversuch aber schlägt fehl, und zwar deswegen, weil diese Beziehung zur Außenwelt nicht über den normalen Weg zustande gebracht wird, sondern durch einen beson-

11 Ders., Totem und Tabu, in: Studienausgabe, Bd. IX, Berlin 1989, 363.

12 Ders., Psychoanalytische Bemerkungen über einen autobiographisch beschriebenen Fall von Paranoia, in: Studienausgabe, Bd. VII, 193. 
deren Mechanismus, nämlich den der Projektion innerer Seelenvorgänge in die Außenwelt. Was diese Projektion genau ist und wie sie genau funktioniert, bleibt in Freuds Werk eine unbeantwortete Frage. Halten wir aber fest: Es geht um einen Heilungsversuch, und zwar im Sinne einer Rekonstruktion einer Beziehung zur Außenwelt.

Zweitens, der Projektionsmechanismus ist ein primitiver Mechanismus, der sich nicht nur in der Paranoia bzw. Psychose findet, sondern allen menschlichen Sinneswahrnehmungen unterliegt und der also „an der Gestaltung unserer Außenwelt normalerweise den größten Anteil hat" ${ }^{“ 13}$ Was der Mechanismus konkret produziert, ist eine, Weltanschauung', und diese Weltanschauung hat im Grunde genommen zwei Merkmale: Erstens, die verschiedenen Personen und Dinge in der Welt werden miteinander in Verbindung gebracht. Es entsteht so eine Ordnung der Dinge. Und, zweitens, diese Ordnung ist transparent und erkennbar. In der Weltanschauung sind also alle Elemente der Außenwelt in eine transparente, erkennbare Ordnung und miteinander in Verbindung gebracht. ${ }^{14}$ Rekonstruktion, Transparenz und Ordnung - als dritter Aspekt kann noch die „Allmacht der Gedanken“ erwähnt werden, d.h. diese Weltanschauung bringt auch die Möglichkeit mit sich, die Außenwelt manipulieren und kontrollieren zu können. Die Beziehung zur Außenwelt ist also inhärent mit der Erkenntnis der Außenwelt verbunden oder findet über Erkenntnis statt, und diese ist inhärent verbunden mit der Möglichkeit, Kontrolle und Macht auszuüben. Ordnung, Erkenntnis und Macht sind also die Kernaspekte des paranoischen Komplexes, die mit einer philosophischen Systembildung in Verbindung gebracht werden können. Und der aufmerksame Zuhörer, der etwas von der modernen Philosophie mitbekommen hat, hat wahrscheinlich schon eine Assoziation; denn hört sich das nicht wie Friedrich Nietzsche oder Michel Foucault und deren Kritik an den großen philosophischen Systemen des Abendlandes an? In Verbindung mit der Philosophie selbst ist also dieser Zugang fruchtbar zu machen für unser Denken

Man sieht also, wie man die Psychoneurosen oder zentrale Aspekte dieser Neurosen in ihrem Zusammenhang, d.h. als Komplexe (als assoziativ miteinander verknüpfte psychische Dynamiken und Konstellationen) zum Muster nehmen kann, um Kenntnis von bestimmten kulturellen Phänomenen zu gewinnen. Wenn wir von Freuds oben zitierter Passage aus „Totem und Tabu" ausgehen, ließe sich eine pathoanalytische Perspektive auf kulturelle Phänomene weiterentwickeln. Die Grundannahme wäre, dass die verschie-

13 Ders., Totem und Tabu, 354.

14 Man muss hier nicht unbedingt an die Weltanschauung eines Paranoikers denken, man könnte auch auf die Weltanschauung des kleinen Hans verweisen, eine infantile Weltanschauung, in der alle Personen, Tiere und Dinge kategorisiert sind in Beziehung zum „Wiwimacher“. In Hans' Weltanschauung haben alle Personen und Tiere Wiwimacher. Die Wiwimacher sind das Kriterium, wonach Hans seine Welt ordnet und in seinem Zusammenhang kennt, vgl. ders., Analyse der Phobie eines fünfjährigen Knaben, in: Studienausgabe, Bd. VIII, Berlin 1989, 92-93. 
denen Psychoneurosen durch unterschiedliche psychische Komplexe (d.h. assoziativ miteinander verbundene seelische Vorgänge) und Mechanismen charakterisiert sind und sich als solche mit unterschiedlichen kulturellen Phänomenen in Verbindung setzen lassen. So wie die Hysterie uns die Bausteine der menschlichen Sexualität zeigt und so wie die Zwangsneurose uns in das komplexe seelische Gewebe von Aggressivität, Ambivalenz, Schuld und Objektbeziehung einführen kann und so wie die Paranoia uns etwas Wesentliches über die seelischen Vorgänge hinter und in Weltanschauungen zeigen kann, so können Hysterie, Zwangsneurose und Paranoia auf besondere Art Kenntnis über bestimmte kulturelle Phänomene bringen.

Über die Hysterie kann man also Kunst verstehen, aber auch die Religion oder die Philosophie? Freud zieht diese Möglichkeiten nicht in Betracht. Das hat schwerwiegende Folgen, die in „Totem und Tabu“ klar ersichtlich werden. Freud wird sich nur jenen Aspekten der Religion widmen, die mit der Zwangsneurose und teilweise auch mit der Phobie in Verbindung gesetzt werden können. Insofern es den paranoischen Mechanismus betrifft, wird Freud ihn ausschließlich mit dem Animismus in Verbindung bringen, und er wird explizit dazu sagen, dass der Animismus im Grunde genommen keine Religion sei, sondern eben nichts mehr oder weniger als eine Weltanschauung. Dass eine Religion auch eine theologische Systematik und Weltanschauung mit sich bringt, gerät so bei ihm völlig außer Sicht. Es gibt m. E. aber kein Argument, diesen Aspekt der Religion nicht mit der Paranoia in Verbindung zu bringen. Ähnliches trifft auf die Beziehung zur Hysterie zu. Jene Aspekte der Religion, die Freud mit der Hysterie in Verbindung hätte bringen können, spielen in „Totem und Tabu“ überhaupt keine Rolle. Man denke hier zum Beispiel an religiöse Ekstase, Mystik, Besessenheit, Visionen, Stigmata, Konvulsionen usw. Freuds Lehrer, Jean-Martin Charcot, hatte Freud diesen Weg eigentlich schon fast vorgezeichnet, indem Charcot und seine Mitarbeiter gerade die Hysterie als Muster genommen hatten, um solche religiösen Phänomene zu deuten. ${ }^{15}$ Freud wird dies nicht machen und erstaunlicherweise zehn Jahre später, 1923, in seiner Studie über die Teufelsneurose im siebzehnten Jahrhundert sogar behaupten:

„Mehrere Autoren, voran Charcot, haben bekanntlich in den Darstellungen der Besessenheit und Verzückung, wie sie uns die Kunst hinterlassen hat, die Äußerungsformen der Hysterie agnosziert; es wäre nicht schwer gewesen, in den Geschichten dieser Kranken die Inhalte der Neurose wiederzufinden, wenn man ihnen damals mehr Aufmerksamkeit geschenkt hätte. ${ }^{\text {"16 }}$

In der Tat wird Freud Christoph Haitzmanns Konvulsionen, Lähmungen, Visionen und Sprachstörungen nicht als Hysterie deuten, sondern als Ausdruck

15 Herman Westerink, Demonical Possession and the Historical Construction of Melancholy and Hysteria, in: History of Psychiatry 25 (2014/3), 335-349.

16 Freud, Eine Teufelsneurose im siebzehnten Jahrhundert, in: Studienausgabe, Bd. VII, Berlin 1989, 287. 
einer Ambivalenz der Gefühle und den Vaterkomplex so, wie man ihn in der Zwangsneurose vorfindet. ${ }^{17}$

Um zu verstehen, warum Freud hier die Hysterie so radikal ausblendet, müssen wir noch einmal zurück zu „Totem und Tabu“. Die Passage am Schluss der zweiten Abhandlung über die verschiedenen Neurosen und deren Verbindung mit kulturellen Phänomenen ist ein Hinweis, in welcher Richtung die pathoanalytische Perspektive ausgearbeitet hätte werden können. Zwei Entwicklungen in Freuds Theoriebildung werden diese Vorgangsweise aber verhindern, und obwohl Freud immer wieder auch die pathoanalytische Perspektive hervorheben wird - und er muss das tun, um der psychoanalytischen Deutung kultureller Phänomene Plausibilität zu verschaffen! - diese Perspektive wird nie in ihrem vollen Potenzial zur Entwicklung gebracht.

\section{Die angewandte Pathoanalyse - ein gescheitertes Projekt?}

Welches sind die theoretischen Entwicklungen, die Freud daran hindern werden, die Möglichkeiten einer pathoanalytischen Perspektive systematisch zu explorieren? M.E. gibt es zwei elementare Entwicklungen in der Theoriebildung, die eine Antwort auf diese Frage bieten. Die erste Entwicklung kann man mit Freuds Theorie über Sexualität verbinden. 1905, in der Erstausgabe der „Drei Abhandlungen“, hatte er die infantile Sexualität aus der Perspektive der Hysterie und der Perversionen radikal als autoerotisch und objektlos beschrieben: „Der Geschlechtstrieb sei also im Kindesalter nicht zentriert und zunächst objektlos, autoerotisch. "18 In der Pubertät wird dann die Sexualität völlig neugestaltet, nämlich verbunden mit einer Funktionalität, die Freud im Blick auf die infantile Sexualität radikal abgewiesen hatte. Die Sexualität wird normalerweise geformt in Beziehung auf ein heterosexuelles Objekt mit dem letztendlichen Ziel der Fortpflanzung: „Die Normalität des Geschlechtslebens wird nur durch das exakte Zusammentreffen der beiden auf Sexualobjekt und Sexualziel gerichteten Strömungen [...] gewährleistet. Der Sexualtrieb stellt sich jetzt in den Dienst der Fortpflanzungsfunktion. "19 Im Grunde genommen hat Freud 1905 also eine Theorie zur Sexualität entwickelt, in der zwei Regimen der Sexualität deutlich voneinander unterschieden werden, nämlich die infantile und die erwachsene Sexualität. Das Grundproblem für Freud ist jetzt, wie man beide Sexualitäten miteinander in Verbindung bringt. Die Frage wird sich zuspitzen auf die Problematik des Objekts. In der Fallstudie über den kleinen Hans wird Freud entdecken, dass es auch in der frühen Kinderzeit schon eine Objektwahl gibt, und zwar nicht über den Sexualtrieb, sondern

17 Ebd., $298 \mathrm{ff}$.

18 Ders., Drei Abhandlungen zur Sexualtheorie, 136. Siehe dazu auch Arnold Davidson, The Emergence of Sexuality. Historical Epistemology and the Formation of Concepts, Cambridge 2001; Philippe Van Haute/Herman Westerink, Hysterie, Sexualität und Psychiatrie.

19 Ders., Drei Abhandlungen zur Sexualtheorie, 112. 
über den Wisstrieb. ${ }^{20}$ Der nächste Schritt ist aber erst wirklich entscheidend. Ab circa 1910 wird Freud die Beziehung zwischen infantiler und erwachsener Sexualität mehr und mehr systematisch in einem entwicklungspsychologischen Modell denken. Die Einführung des Narzissmus-Begriffes ist hier ein entscheidender Schritt. Er fängt jetzt an, die Sexualität als eine Sexualentwicklung zu beschreiben, und unterscheidet unterschiedliche Stufen: Autoerotik, Narzissmus, homosexuelle Objektwahl, heterosexuelle Objektwahl. ${ }^{21}$

Die Konsequenz dieser methodischen Verschiebung in Richtung eines entwicklungspsychologischen Ansatzes unterminiert auf fundamentale Weise die vorherige pathoanalytische Perspektive. Diese Perspektive stützte sich nämlich, wie wir gesehen haben, auf die Prämisse eines Kontinuums von Pathologie und Normalität. Erstere sei nur eine Vergrößerung und Vergröberung der zweiten. Das ist aber in einem entwicklungspsychologischen Ansatz nicht mehr der Fall. Ein Entwicklungsmodell ist inhärent normativ, indem es immer und prinzipiell eine normale Entwicklung beschreibt, anhand derer die Abirrungen definiert werden können. Freuds Ansatz wird somit zunehmend heteronormativ. Denn jetzt gilt Folgendes: Normalerweise entwickelt sich die Sexualität in die Richtung einer heterosexuellen Objektwahl. Alles andere ist Abirrung, Störung und abnorm. Das Verhältnis zwischen Pathologie und Normalität ändert sich hier grundliegend: Die Normalität kann wieder klar mit der Pathologie kontrastiert werden. Statt Kontinuum gibt es also erneut den Kontrast. Die Auswirkungen dieser Vorgangsweise kann man sehr gut in Freuds „Die Zukunft einer Illusion“ sehen. Dort wird Religion anders als es vorher noch in „Totem und Tabu“ der Fall war - nicht primär aus einer pathoanalytischen Perspektive betrachtet, sondern aus einer entwicklungspsychologischen Perspektive, die es Freud jetzt ermöglicht, die Religion als ein überholtes Stadium in der Kulturgeschichte zu betrachten und sie nur noch als illusionäres Gegenüber zum Aufschwung der Wissenschaft und zur „Erziehung zur Realität“ darzustellen. Den Ansatz für diese Perspektive finden wir schon in „Totem und Tabu“, wo Freud die Stufen der Sexualentwicklung (Autoerotismus und Narzissmus; Objektfindung; Reifezustand und Anpassungsfähigkeit des Erwachsenen) zum ersten Mal mit der Evolution der Kultur und der Weltanschauungen in Verbindung bringt (animistische Phase; religiöse Phase; wissenschaftliche Phase). ${ }^{22}$

20 Ders., Analyse der Phobie eines fünfjährigen Knaben, 111-118; vgl. ders., Über infantile Sexualtheorien, in: Studienausgabe, Bd. V, Berlin 1989, $174 \mathrm{ff}$.

21 Ders., Psychoanalytische Bemerkungen über einen autobiographisch beschriebenen Fall von Paranoia, in: Studienausgabe, Bd. VII, Berlin 1989, 184-186; vgl. ders., Drei Abhandlungen zur Sexualtheorie, 103-106; ders., Die Disposition zur Zwangsneurose, in: Studienausgabe, Bd. VII, Berlin 1989, 112-113. In den „Drei Abhandlungen“ sind die Abschnitte über die Entwicklungsphasen der sexuellen Organisation und über die zweizeitige Objektwahl erst 1915 im Text eingefügt.

22 Ders., Die Zukunft einer Illusion, in: Studienausgabe, Bd. IX, 168-178; ders., Totem und Tabu, 376, 378 . 
Noch eine zweite entscheidende theoretische Entwicklung muss erwähnt werden. Sie betrifft den Schritt von der Zerrbild-Passage in der zweiten Abhandlung (geschrieben 1912) bis zur vierten Abhandlung, wo Freud den Kernkomplex der Zwangsneurose, den Ödipuskomplex, zum Kernkomplex aller Neurosen überhaupt proklamiert: Die psychoanalytische Forschung zeigt uns, dass der Ödipuskomplex „den Kern aller Neurosen bildet, soweit sie bis jetzt unserem Verständnis nachgegeben haben“ ${ }^{23}$ Das bedeutet nicht nur, dass die Hysterie und die Paranoia ab jetzt aus der Perspektive der Zwangsneurose betrachtet werden müssen und somit auch in dieser Richtung interpretiert werden sollten; darüber hinaus bedeutet es auch, dass der implizite Anspruch in der Zerrbild-Passage jetzt verneint wird. Er zielte immerhin implizit darauf, man könne die unterschiedlichen Psychoneurosen und Komplexe als unterschiedliche Muster nehmen, um unterschiedliche kulturelle Phänomene zu analysieren. Der unterscheidende Aspekt fällt jetzt aber weg. Das erklärt auch, warum später Haitzmanns Teufelsneurose nicht mehr aus Sicht der Hysterie gedeutet werden kann oder muss, sondern direkt mit dem Kernkomplex in Verbindung gebracht wird. Auch diese Entwicklung impliziert eine grundlegende Unterminierung eines pathoanalytischen Zugangs. Noch dazu sind die Bestimmung des Kernkomplexes der Neurosen und die Hinwendung zum entwicklungspsychologischen Ansatz eng miteinander verknüpft. Dem Ödipuskomplex wird eine zentrale Rolle beigemessen, wenn es um die normale Gestaltung des Seelenlebens geht.

Man kann nun fragen, warum diese Entwicklung ein Problem darstellt. Warum sollten wir dieses Schibboleth der Psychoanalyse in Frage stellen, wenn wir es doch immer wieder klinisch bestätigt finden? Die Frage ist aber, ob diese Entwicklung in der Freud'schen Theorie dazu beigetragen hat, dass die Psychoanalyse die Religion besser verstehen und plausibler deuten kann. Nehmen wir mal folgendes Beispiel. Dass Christus, der Sohn des erhöhten Vaters, eine zentrale Rolle im Christentum spielt, ist evident, und dass hierin eine ödipale Struktur sichtbar wird, scheint plausibel. Aber um die verschiedenen Frömmigkeiten zu verstehen, genügt das nicht. Es macht freilich schon einen Unterschied, ob man sich mit Christus in seinem Leiden identifiziert und darin vielleicht sogar einen gewissen Ekel überwindet oder ob man sich in Beziehung zu Christus oder Gott schuldig fühlt wegen der Entfremdung und des Verlustes der Anwesenheit Gottes oder ob man sich schuldig fühlt, weil man Gottes Wille nicht gehorsam war und seine Gesetze übertreten hat oder ob man die persönliche Beziehung zu Christus als Kriterium für eine Unterscheidung zwischen Frommen und Ungläubigen nimmt. Die vier Optionen korrespondieren mit den vier psychopathologischen Komplexen: Hysterie, Melancholie, Zwangsneurose und Paranoia.

Die Pathoanalyse kann für uns eventuell von großer Bedeutung sein, gerade um die unterschiedlichen Aspekte einer Religion besser verstehen zu können.

23 Ebd., 439.

Wege zum Menschen, 69. Jg., 301-311, ISSN: 0043-2040 (print), 2196-8284 (online) (C) 2017 Vandenhoeck \& Ruprecht GmbH \& Co. KG, Göttingen 
Das könnte z.B. ein sehr interessanter Zugang sein, die unterschiedlichen christlichen Konfessionen, die in der Neuzeit in ihrer Hinwendung zum Subjekt entstehen, zu analysieren. Anders gesagt, eine pathoanalytische Perspektive könnte bei der psychologischen Erforschung unterschiedlicher Frömmigkeitstypen hilfreich sein.

Dr. Herman Westerink, Titus Brandsma Institut/Center for Contemporary European Philosophy, Radboud Universität, 6500 HD Nijmegen, Niederlande; E-Mail: h.westerink@ftr.ru.nl 